\title{
Capacidade funcional e dor em idosos nos períodos pré e pós-operatório de cirurgia cordíaca
}

\section{Functional capacity and pain in the elderly prior to and after cardiac surgery}

\author{
Kelin Gnoatto'; Josiéle Casanova Matteii; Alana Piccoli²; Janaíne Cunha Polese ${ }^{3}$; Silvia Lanziotti \\ Azevedo da Silva3; Rodrigo Costa Schuster4; Camila Pereira Leguisamo ${ }^{5}$ \\ ${ }^{1}$ Fisioterapeuta - UPF/RS. Passo Fundo, RS - Brasil \\ ${ }^{2}$ Fisioterapeuta Residente - Residência Integrada em Saúde, Grupo Hospitalar Conceição - GHC/RS. Porto Alegre, RS - Brasil. \\ ${ }^{3}$ Fisioterapeuta, Doutoranda em Ciência da Reabilitação - UFMG/MG. Belo Horizonte, MG - Brasil. \\ ${ }^{4}$ Fisioterapeuta, Mestre em Ciências Médicas - UFRGS/RS. Porto Alegre, RS - Brasil. \\ ${ }^{5}$ Fisioterapeuta, Doutora em Ciências da Saúde - IC-FUC/RS. Porto Alegre, RS - Brasil. \\ Endereço para correspondência \\ Rodrigo Costa Schuster \\ R. Bento Gonçalves, 2859/502, São Pelegrino \\ 95020-412 - Caxias do Sul - RS [Brasil] \\ rodrigo.schuster@fsg.br
}

\begin{abstract}
Resumo
Introdução: A cirurgia cardíaca pode gerar dor e perdas funcionais em idosos. Objetivo: Avaliar capacidade funcional e dor de idosos em períodos pré e pós-operatório (PO). Métodos: Realizou-se um estudo longitudinal, com amostra de 46 idosos submetidos à cirurgia cardíaca. Utilizaram-se Medida de Independência Funcional (MIF), Escalas Visual Analógica e Numérica da Dor e Diagrama Corporal, avaliados em três momentos. Usaram-se o teste de Wilcoxon para comparar a dor; e o de Spearman, para avaliar as associações entre as variáveis estudadas. Resultados: A média de idade foi $69,3 \pm 1$ anos, prevalecendo o sexo masculino. Observou-se independência em $97,8 \%$ da amostra no pré-operatório; $65,2 \%$, dependência de até $50 \%$, no segundo dia PO; e $60,9 \%$, dependência de até $75 \%$, no quinto dia PO. A associação entre tempo de internação total, pósoperatório e MIF mostrou-se significativa no segundo e quinto PO. Conclusão: Idosos submetidos à cirurgia cardíaca apresentam declínio da funcionalidade e diminuição da dor com o passar dos dias.
\end{abstract}

Descritores: Cirurgia torácica; Dor; Fisioterapia; Idoso.

\begin{abstract}
Introduction: Cardiac surgery can cause pain and disability in the elderly. Objective: To evaluate functional capacity and pain experienced in the elderly before and after surgery (PO). Methods: A longitudinal study was carried out with a sample of 46 elderly patients undergoing cardiac surgery. We used the Functional Independence Measure (FIM), both the Visual Analog Scale (VAS) and Numerical Rating Scale (NRS) for evaluating pain and Body Diagrams, at three separate moments. The Wilcoxon test was used for comparing the pain and Spearman's for evaluating associations between studied variables. Results: The mean age of the subjects (males, in the majority) was $69.3 \pm 1$ years. Independence was observed in $97.8 \%$ of the sample in the preoperative period, while $65.2 \%$ showed dependence of up to $50 \%$ in the second postoperative day and $60.9 \%$ up to $75 \%$ dependence on the fifth postoperative day. The association between total length of hospital stay, postoperative period and MIF was significant in the second and fifth postoperative day. Conclusions: Elderly patients undergoing cardiac surgery showed an increase in disability and a decrease in pain experienced with each passing day.
\end{abstract}

Key words: Aged; Pain; Physical therapy; Thoracic surgery. 


\section{Introdução}

Com o crescente aumento da população idosa, o envelhecimento é hoje um fenômeno universal. O elevado número de idosos já pode ser percebido em todo o Brasil e, segundo a Organização Mundial de Saúde (OMS), até o ano de 2025, a população idosa brasileira crescerá 16 vezes, enquanto observa-se que mundialmente esse número aumentará em cinco vezes ${ }^{1}$, classificando o país como a sexta população do mundo em idosos, com aproximadamente 34 milhões de indivíduos ${ }^{2}$.

A capacidade funcional é um importante marcador de saúde em idosos ${ }^{3}$, e a função é definida pela capacidade que o indivíduo tem de adaptar-se aos problemas cotidianos e suas atividades ${ }^{4}$. Na prática, trabalha-se com os conceitos de capacidade e incapacidade funcional para gestos diários, desde a dificuldade até a impossibilidade de realizá-los ${ }^{5}$. Os idosos são mais suscetíveis a complicações causadas pelo repouso prolongado no leito durante a hospitalização ${ }^{6}$, o que poderia acarretar alterações na funcionalidade. Em um estudo é reportado que mulheres idosas que foram hospitalizadas apresentaram maior chance de desenvolver incapacidade para Atividades de Vida Diária (AVD), quando comparadas a idosas que não necessitaram permanecer em hospitais?.

Nos últimos anos, houve um aumento no número de procedimentos cirúrgicos em pacientes idosos $^{8}$. A cirurgia cardíaca é um procedimento complexo que implica na alteração de vários mecanismos fisiológicos; no contato com medicamentos e materiais que podem ser nocivos ao organismo e ainda em um grande estresse orgânico?.

A incapacidade é comum nos indivíduos idosos ${ }^{5}$, devido às próprias características inerentes ao envelhecimento, podendo ser ainda agravada pela hospitalização ${ }^{7}$. Nesse sentido, com o aumento da prevalência da população idosa, surge também a necessidade de instrumentos para avaliação da capacidade funcional ${ }^{10}$. A dor, por sua vez, é considerada um dos principais fatores que explicam os altos níveis de inabilidade funcional do indivíduo idoso, pois limita suas atividades ${ }^{11}$. A dor em idosos é um preocupante problema de saúde pública, necessitando de mensurações e avaliações acuradas, porém subjetivas, minimizando a morbidade e melhorando a qualidade de vida dessa população. No entanto, é reportado que instrumentos de avaliação e mensuração raramente são usados para monitorar a dor e incapacidade funcional de idosos ${ }^{12}$.

Nesse escopo, esforços devem ser feitos no sentido de entender e maximizar parâmetros relacionados à avaliação da funcionalidade e dor do paciente, pois identificando seu declínio funcional e os fatores associados, a reabilitação auxiliará no desenvolvimento e manutenção de um nível desejável das condições físicas, assegurando o retorno do indivíduo a uma vida ativa e produtiva da melhor maneira possível ${ }^{13,14}$.

Assim, os objetivos deste estudo foram avaliar a capacidade funcional de idosos nos períodos pré e pós-operatório de cirurgia cardíaca, além de avaliar e comparar a dor aos esforços nos períodos pré-operatório e segundo e quinto pósoperatórios. Além disso, procurou-se verificar a associação entre a dor e a pontuação na escala Medida de Independência Funcional (MIF), entre tempo de internação e incapacidade funcional.

\section{Materiais e métodos}

Trata-se de um estudo longitudinal em que se avaliou a capacidade funcional e dor de pacientes idosos submetidos à cirurgia cardíaca nos períodos pré e pós-operatório, no Hospital São Vicente de Paulo, na cidade de Passo Fundo (RS).

A amostra foi composta por 46 idosos, que foram amostrados por conveniência, de ambos os sexos submetidos à cirurgia cardíaca eletiva, internados no Centro de Terapia Intensiva Cardiológico e/ ou em leitos hospitalares. Os tipos de cirurgias cardíacas realizadas foram para correção de revascularização do miocárdio, prótese mitral, prótese aórtica, aneurisma, dis- 
secção de aorta, Comunicação Intraventricular (CIV) e para Comunicação Intra-atrial (CIA).

Os critérios de exclusão do estudo foram pacientes que possuíssem idade inferior a 60 anos, apresentassem déficit neurológico ou cognitivo, que desistissem de participar do estudo e pacientes que fossem a óbito durante o período da coleta de dados. Inicialmente, foram selecionados 53 indivíduos, e desses, sete foram excluídos: três por terem desistido; e quatro, por óbito.

Após aprovação da pesquisa pelo Comitê de Ética da Universidade de Passo Fundo e assinatura do Termo de Consentimento Livre e Esclarecido pelo paciente, conforme as orientações da Resolução 196/96 do Conselho Nacional de Saúde, foram coletados dados por meio do preenchimento de uma ficha que continha os dados de identificação do participante, tais como nome, profissão, data de nascimento, idade, procedência, endereço, sexo, peso, altura, índice de massa corporal (IMC), número do registro e leito, data de internação, procedimento cirúrgico, uso de circulação extracorpórea (CEC), e fatores de risco (tabagismo e etilismo). Os critérios utilizados para classificar tabagistas e etilistas foram: (1) pacientes que fumam, bebem ou fumaram, (2) beberam até 30 dias antes da operação e (3) ex-tabagistas e ex-etilistas com história prévia, anterior a 30 dias da avaliação.

Em seguida, foi aplicado o instrumento MIF a fim de avaliar a capacidade funcional dos idosos avaliados ${ }^{16}$. Logo após, aplicaramse a Escala Visual Analógica da Dor ${ }^{16}$, a Escala Numérica da Dor $^{17}$ e o Diagrama Corporal ${ }^{18}$ como instrumentos para mensurar o grau e a localização de dor em esforços, nos períodos pré e pós-operatório de cirurgia cardíaca.

Os idosos participantes foram submetidos à Escala Visual Analógica da Dor (EVA) que consiste em uma linha não graduada cujas extremidades correspondem à ausência de dor, em geral situada na extremidade esquerda; e à pior dor imaginável, na extremidade oposta ${ }^{16}$. Em seguida, aplicou-se a escala Numérica da Dor, em que o paciente é informado sobre a necessidade de classificar sua dor em notas que variam de 0 a 10, de acordo com a intensidade da sensação. Nota zero corresponderia à ausência de dor, enquanto nota 10 , a maior intensidade imagináve ${ }^{17}$. E, por último, utilizou-se o Diagrama Corporal, que consiste em um desenho em forma de linhas do corpo de frente e de costas, no qual o idoso indicava a localização da dor ${ }^{18}$.

A MIF foi utilizada com o intuito de fazer uma análise que quantifica a carga de cuidados demandada por uma pessoa para a realização de uma série de tarefas motoras e cognitivas ${ }^{15}$ Esse instrumento contém duas dimensões, sendo elas as motoras: autocuidados, controle de esfíncteres, transferências, locomoção; e as cognitivas: comunicação e cognição social. Avalia 18 tarefas pontuadas de 1 (dependência total) a 7 (independência total), assim seu escore total pode variar de 18 a 126 pontos, e quanto menor a pontuação, maior o grau de dependência ${ }^{15,19}$.

Para avaliar a perda funcional (PF\%), utilizou-se a equação proposta por Borges et al. ${ }^{20}$

$\mathrm{PF} \%=\frac{\begin{array}{c}\text { (escore funcional } \\ \text { momento inicial }- \text { escore } \\ \text { funcional momento final) }\end{array}}{\begin{array}{l}\text { (escore funcional } \\ \text { momento inicial) }\end{array}} \times 100 \%$

As avaliações foram realizadas sempre pelo mesmo avaliador e no mesmo turno. Todos os pacientes recebiam atendimento fisioterapêutico apenas pela equipe do hospital, duas vezes ao dia, sem nenhuma intervenção dos avaliadores deste estudo. Os dias de internação total foram contados a partir do primeiro dia de internação do paciente até o da alta hospitalar; e os dias de internação pós-cirúrgicos, a partir do primeiro dia após a cirurgia cardíaca até o dia da alta hospitalar.

\section{Anólise estatística}

As variáveis foram descritas como média \pm desvio-padrão ou como mediana (diferença interquartílica), quando contínuas; e frequência absoluta e relativa, quando categóricas. Utilizou-se o teste de 
Kolmogorov-Smirnov para avaliar a hipótese de aderência à normalidade das variáveis contínuas. E utilizaram-se o teste de Wilcoxon para comparar a dor entre os diferentes momentos de aferição e o teste de correlação de Spearman para avaliar a hipótese de correlação entre dor e pontuação da MIF e a mesma pontuação da MIF e tempo de internação, devido à violação do pressuposto de normalidade apresentada por essas variáveis.

\section{Resultados}

Os indivíduos em estudo tinham variação de idade entre 60 e 86 anos, com média de 69,3 $\pm 0,9$ anos, prevalecendo o sexo masculino em 25 (54,3\%) pacientes. Em relação ao peso, altura e IMC os valores correspondentes da média foram $68,9 \pm 1,5$ $\mathrm{kg} ; 1,6 \pm 0,1 \mathrm{~cm} ; 25,5 \pm 0,6 \mathrm{~kg} / \mathrm{m}^{2}$, respectivamente. Os procedimentos cirúrgicos realizados foram a revascularização do miocárdio, totalizando $63,0 \%$; prótese aórtica $21,7 \%$; prótese mitral $10,9 \%$, e outros $4,3 \%$. Na maioria dos pacientes foi utilizada a CEC $(95,6 \%)$. A caracterização da amostra está sumarizada na Tabela 1.

\section{Tabela l: Características da amostra}

\begin{tabular}{cc}
\hline Característica & $\mathbf{N}=\mathbf{4 6}$ \\
\hline Idade (anos) & $69,3 \pm 0,9$ \\
Sexo & \\
Masculino(\%) & $25(54,3)$ \\
Peso (kg) & $68,9 \pm 1,5$ \\
Altura (m) & $1,6 \pm 0,1$ \\
IMC (kg/m2) & $25,5 \pm 0,6$ \\
Fatores de risco & \\
Tabagismo (\%) & 2,2 \\
Tipo de cirurgia & \\
RVM (\%) & 63,1 \\
Outras (\%) & 36,9 \\
CEC & \\
Sim (\%) & 95,6 \\
\hline
\end{tabular}

Os dados referentes à intensidade da dor podem ser observados na Figura 1. Observouse diferença estatisticamente significativa entre o período pré-operatório e o segundo dia pós- operatório, e o pré-operatório e o quinto dia pós-operatório, e segundo e quinto dias de pósoperatórios $(\mathrm{p}<0,001)$.

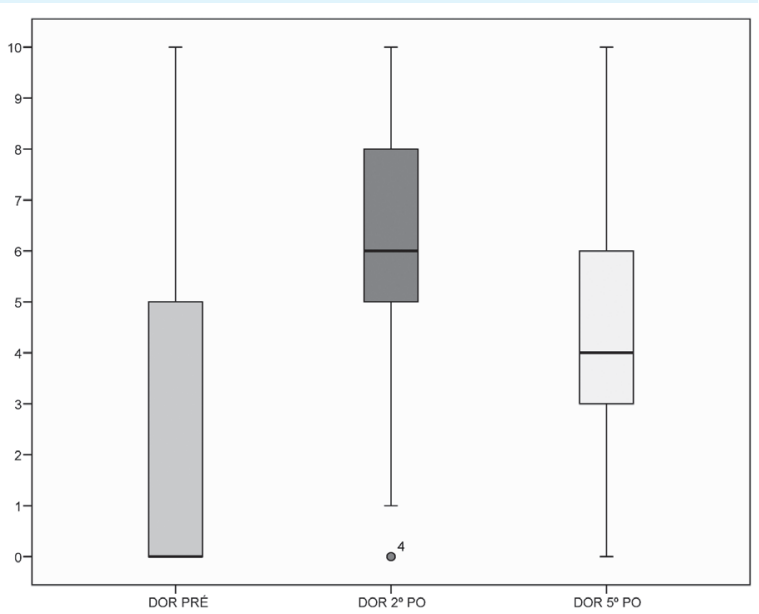

Figura 1: Comparação da intensidade da dor nos períodos pré-operatório e segundo e quinto pós-operatório avaliado pela EVA

Segundo a Escala Numérica da Dor, no período pré-operatório classificou-se a dor como fraca $(2,6 \pm 3,6)$; no segundo pós-operatório, como forte $(6,1 \pm 2,5)$; e no quinto pós-operatório, como moderada $(4,1 \pm 2,4)$. O local de dor mais relatado pelos pacientes no pré-operatório foi na região do tórax anterior à esquerda $(36,9 \%)$, sendo característica de angina; no segundo dia pósoperatório, no qual se relatou algia na região do esterno (91,3\%); e ao quinto dia pós-operatório, em que os pacientes queixaram-se de algia também na região esternal (86,9\%), sendo essa dor pós-operatória decorrente ao trauma cirúrgico.

$\mathrm{Na}$ caracterização da funcionalidade pela MIF total no pré-operatório, 45 (97,8\%) pacientes apresentaram independência completa. No segundo dia pós-operatório 30 (65,2\%) voluntários mostraram dependência de até $50 \%$, e nenhum paciente apresentou independência funcional nesse período. No quinto dia pós-operatório, 28 $(60,9 \%)$ participantes apresentaram dependência de até $75 \%$. Os resultados referentes à capacidade funcional foram significativos do período pré-operatório para o segundo dia pós-operatório, pois houve perda funcional; e ainda se mos- 
traram significativos do pré-operatório para o quinto dia pós-operatório e também no segundo para o quinto dia pós-operatório, apresentando aumento na independência das AVDs.

Avaliou-se a hipótese de associação entre a dor e a MIF total, a motora e a cognitiva, observando-se associação estatisticamente significativa entre dor no quinto PO e MIF motora no quinto PO (Tabela 2).

A média do tempo de internação hospitalar total foi $15,1 \pm 9,2$ dias. A correlação entre MIF total, MIF motora e MIF cognitiva com o tempo de internação hospitalar total e o pósoperatório, foi estatisticamente significativa entre MIF total e motora no segundo e quinto PO e tempo de internação total e período pósoperatório (Tabela 3).

\section{Discussão}

O predomínio do sexo masculino na amostra foi semelhante ao do estudo de Silva, Nascimento e Viotti ${ }^{21}$, no qual $65,8 \%$ da amostra eram homens. A revascularização do miocárdio foi a cirurgia realizada com mais frequência pelos participantes, assim como observado no estudo de Loures et al. ${ }^{22}$, no qual a revascularização do miocárdio apresentou maior frequência em relação aos outros procedimentos, totalizando $66,6 \%$.

A presença de dor foi constante durante todo o período do estudo, sendo mais intensa no segundo dia pós-operatório e relatada como localizada na região esternal. Concordando com os achados do trabalho aqui apresentado, Mueller et al. ${ }^{16}$, observaram, em seu estudo, que mais de $50 \%$ dos pacientes ainda apresentavam dor na região da esternotomia no sétimo dia após a cirurgia cardíaca, e que a dor era maior no segundo dia de PO.

Em relação à dor referida pelos pacientes na região da esternotomia, ela poderia ser atribuída à extensão da incisão ${ }^{23}$ e fricção do esterno pela instabilidade do tórax superior ${ }^{24}$. Todos os pacientes saíram do bloco cirúrgico com dreno de
Tabela 2: Correlação entre dor e MIF

\begin{tabular}{ccc}
\hline & Correlação & $\mathrm{p}^{*}$ \\
\hline Dor e MIF Total Pré & 0,21 & 0,162 \\
Dor e MIF Motora Pré & 0,22 & 0,148 \\
Dor e MIF Cognitiva Pré & 0,19 & 0,200 \\
Dor e MIF Total $2^{\circ}$ PO & $-0,04$ & 0,820 \\
Dor e MIF Motora $2^{\circ}$ PO & 0,06 & 0,685 \\
Dor e MIF Cognitiva $2^{\circ}$ PO & $-0,08$ & 0,611 \\
Dor e MIF Total 5 $5^{\circ}$ PO & $-0,28$ & 0,057 \\
Dor e MIF Motora 5 $5^{\circ}$ PO & $-0,29$ & $0,048^{*}$ \\
Dor e MIF Cognitiva 5 $5^{\circ}$ PO & $-0,191$ & 0,204
\end{tabular}

* Coeficiente de Correlação de Spearman

**Nível de significância $p \leq 0,05$

MIF: medida de independência funcional

Tabela 3: Correlação entre MIF e tempo de internação

\begin{tabular}{|c|c|c|c|c|}
\hline \multirow[b]{2}{*}{$\begin{array}{l}\text { Medida de } \\
\text { Independência } \\
\text { Funcional }\end{array}$} & \multicolumn{2}{|c|}{$\begin{array}{c}\text { Tempo de } \\
\text { Internação } \\
\text { Total }\end{array}$} & \multicolumn{2}{|c|}{$\begin{array}{c}\text { Tempo de } \\
\text { Internação } \\
\text { Pós-Operatório }\end{array}$} \\
\hline & $\begin{array}{c}\text { Coeficiente } \\
\text { de } \\
\text { Correlação }\end{array}$ & $\mathbf{p}^{*}$ & $\begin{array}{c}\text { Coeficiente } \\
\text { de } \\
\text { Correlação }\end{array}$ & $\mathbf{p}^{*}$ \\
\hline MIF Total Pré & $-0,3$ & 0,849 & $-0,2$ & 0,891 \\
\hline MIF Motora Pré & $-0,07$ & 0,644 & $-0,10$ & 0,529 \\
\hline $\begin{array}{l}\text { MIF Cognitiva } \\
\text { Pré }\end{array}$ & $<0,01$ & 0,977 & 0,14 & 0,364 \\
\hline MIF Total $2^{\circ} \mathrm{PO}$ & $-0,37$ & $0,012^{* *}$ & $-0,29$ & $0,048^{* *}$ \\
\hline $\begin{array}{c}\text { MIF Motora } \\
2^{\circ} \mathrm{PO}\end{array}$ & $-0,37$ & $0,010 * *$ & $-0,32$ & $0,030 * *$ \\
\hline $\begin{array}{l}\text { MIF Cognitiva } \\
2^{\circ} \mathrm{PO}\end{array}$ & $-0,19$ & 0,203 & $-0,12$ & 0,44 \\
\hline MIF Total $5^{\circ} \mathrm{PO}$ & $-0,35$ & $0,017^{* *}$ & $-0,36$ & $0,015^{* *}$ \\
\hline $\begin{array}{c}\text { MIF Motora } \\
5^{\circ} \mathrm{PO}\end{array}$ & $-0,36$ & $0,015^{* *}$ & $-0,34$ & $0,021^{* *}$ \\
\hline $\begin{array}{l}\text { MIF Cognitiva } \\
5^{\circ} \mathrm{PO}\end{array}$ & $-0,13$ & 0,40 & $-0,26$ & 0,079 \\
\hline
\end{tabular}

* Teste de Correlação de Spearman

**Nível de significância $p \leq 0,05$

MIF: medida de independência funcional.

tórax, corroborando os achados de Gacomazzi ${ }^{25}$, justificando a diminuição da funcionalidade nos indivíduos do segundo pós-operatório.

Em relação à redução da funcionalidade observada entre o período pré-operatório e o segundo dia de pós-operatório, constatou-se que essa diminuição ocorre de forma diferenciada entre as atividades avaliadas pela MIF, tanto no 
domínio motor quanto no cognitivo. Quanto ao domínio autocuidado, houve maior perda funcional na categoria Banho, por exemplo. Entre as atividades de Transferências a maior perda funcional apresentou-se na categoria Transferência - Vaso sanitário e Chuveiro; já na Locomoção a categoria Locomoção - Escadas mostrou a maior perda funcional. Essas porcentagens justificamse pelo fato de o paciente estar ainda com dreno de tórax, não podendo locomover-se, e por orientações médicas pós-cirúrgicas. Nesse período, os pacientes recebiam banho no leito, sem realizar qualquer tipo de esforço, e a ida ao banheiro não ocorreu também, pois se encontravam sondados.

Ainda do período pré-operatório para o segundo pós-operatório, a dimensão controle de esfíncter não foi quantificada em relação ao Controle de Urina, pois os pacientes encontravam-se utilizando sondas para controle do balanço hídrico, seguindo as normas do setor; o Controle de Fezes não foi avaliado devido à constipação apresentada por todos os pacientes. Segundo Yardeni ${ }^{26}$, é frequente um balanço hídrico positivo que se inicia na sala de operações e se prolonga pelos primeiros dias pós-operatórios.

A associação significativa entre dor e MIF total e MIF motora no quinto PO pode indicar que pacientes idosos evitam realizar atividades devido à dor. Deve-se ressaltar a proximidade com o nível de 5\% apresentada pela significância da associação entre dor e MIF total no quinto PO. Tais inferências confirmam os achados de Borges et al. ${ }^{20}$, uma vez que o nível de dor teve repercussão significativa na funcionalidade de pacientes submetidos à cirurgia cardíaca.

A perda de funcionalidade após internação hospitalar é comum em pacientes idosos, devido aos diversos motivos de internação. $\mathrm{O}$ estudo de Boyd ${ }^{27}$ evidenciou uma redução de funcionalidade, mesmo após um ano de alta hospitalar, em indivíduos com mais de 70 anos. Natarajan ${ }^{28}$, por sua vez, afirma que a cirurgia cardíaca para idosos com indicação, após a sua recuperação completa, ajuda na melhora da funcionalidade em níveis superiores aos apresentados no período pré-operatório, embora esses sejam ainda menores, quando comparados com indivíduos jovens ${ }^{29}$.

\section{Conclusão}

Idosos submetidos à cirurgia cardíaca apresentam redução na funcionalidade no período pós-operatório, com melhora nos dias consecutivos depois desse procedimento, no entanto sem retornar aos valores iniciais. A dor foi uma queixa que persistiu durante todo o período de internação, observando-se um aumento no pós-operatório recente de cirurgia cardíaca, afetando a funcionalidade dos indivíduos. Nesse sentido, idosos que ficam, por um tempo prolongado, hospitalizados apresentam maior alteração funcional, e a fisioterapia pode atuar objetivando o retorno da capacidade funcional desses indivíduos.

\section{Referências}

1. Esteves B. O Brasil de cabelos brancos. Ciência Hoje. 1998;23(137):18-21.

2. Veras R. Fórum: envelhecimento populacional e as informações de saúde do PNAD: demandas e desafios contemporâneos. Cad Saúde Pública. 2007;23(10):2463-66.

3. Siqueira, AB, Cordeiro, RC, Perracini, MR. Impacto funcional da internação hospitalar de pacientes idosos. Rev Saúde Pública. 2004;38(5):687-94.

4. Caldas CP. Envelhecimento com dependência: responsabilidades e demandas da família. Cad Saúde Pública. 2003;19(3):773-81.

5. Rosa T, Benício M, Latorre O. Fatores determinantes da capacidade funcional em idosos. Rev Saúde Pública. 2003;37(1):40-8.

6. Hirsch $\mathrm{CH}$, Sommers L, Olsen A. The natural history of functional morbid hospitalized older patients. JAGS. 1999;38:1296-303.

7. Boyd C, Xue Q, Guralnik J, Fried LP. Hospitalization and development of dependence in activities of daily living in a cohort of disabled older women: the women's health and aging study I. J Gerontol. 2005;60(7):888-93. 
8. Alfonso F, Azcona L, Perez-Vizcayno MJ, Hernadez. Initial results and long-term clinical and angiographic implications of coronary sting in elderly patients. Am J Cardiology. 1999;83(10):1483-87.

9. Aziz S, Grover FL. Cardiovascular surgery in the elderly. Cardiology Clinics. 1999;17(1):213-31.

10. Duarte $\mathrm{YAO}$, Andrade CL, Lebrão ML. O índice de Katz na avaliação da funcionalidade dos idosos. Rev Esc Enferm USP. 2007;41(2):317-25.

11. Andrade FA, Pereira LV, Sousa FAEM. Mensuração da dor no idoso: uma revisão. Rev Latinoam Enferm. 2006;14(2):271-6.

12. Gold DT, Roberto KA. Correlates and consequences of chronic pain in older adults. Geriatric Nursing. 2000;21(5):270-7.

13. Buchler RDD, Ferraz AS, Meneghelo RS. Princípios gerais e aplicações da reabilitação. Rev Soc Cardiol Estado de São Paulo. 1996;6(1):11-22.

14. Costa CAC, Yazbek J, Sabbag LMS. Alterações eletrocardiográficas e cardiovasculares em pacientes com infarto do miocárdio pregresso submetido a programa de reabilitação cardíaca supervisionado. Acta Fisiátrica. 1997;25:82-9.

15. Riberto M, Miyazaki H, Jucá SSH. Validação da versão brasileira da medida de independência funcional. Acta Fisiátrica. 2004;11(2):72-6.

16. Mueller XM, Tinguely L, Tevaearai HT. Pain location, distribution, and intensity after cardiac surgery. Chest. 2000;118(2):391-6.

17. Marques AP, Assumpção A, Matsutani LA. Fibromialgia e Fisioterapia: avaliação e tratamento. São Paulo: Manole; 2007. p. 16-8.

18. Silva YP, Silva JF, Costa LP. Avaliação da dor na criança. Rev Assoc Méd Minas Gerais. 2004;14(1):S92-S6.

19. Ricci NA, Lemos ND, Orrico KF. Evolução da Independência funcional de idosos atendidos em programa de assistência domiciliária pela óptica do cuidador. Acta Fisiátrica. 2006;13(1):26-31.
20. Borges JBC, Ferreira DLMP, Carvalho SMR. Avaliação de intensidade de dor e da funcionalidade no pós operatório recente de cirurgia cardíaca. Rev Bras Cir Cardiovasc. 2006;21(4):393-402.

21. Silva LHF, Nascimento CS, Viotti LAP. Revascularização do miocárdio em idosos. Rev Bras Cir Cardiovasc. 1997;12(2):132-40.

22. Loudes DRR, Carvalho RG, Mulinari L. Cirurgia cardíaca no idoso. Rev Bras Cir Cardiovasc. 2000;15(1):1-5.

23. Power, I. Recent advances in postoperative pain therapy. Br J Anaesth. 2005;95(1):43-51.

24. Guizilini S, Gomes WJ, Faresin SM. Avaliação da função pulmonar em pacientes submetidos à cirurgia de revascularização do miocárdio com e sem circulação extra corpórea. Rev Bras Cir Cardiovasc. 2005;20(3):310-6.

25. Giacomazzi CM, Lagni VB, Monteiro MBA. Dor pósoperatória como contribuinte do prejuízo na função pulmonar em pacientes submetidos à cirurgia cardíaca. Rev Bras Cir Cardiovasc. 2006;21(4):386-92.

26. Yardeni IZ, Beilin B, Mayburn E. The Effect of perioperative intravenous lidocaine on postoperative pain and immune function. Anesth Analg. 2009;109(5):1464-9.

27. Boyd CM, Landefeld CS, Counsell SR. Recovery of activities of daily living in older adults after hospitalization for acute medical illness. J Am Geriatr Soc. 2008;56(12):2171-9.

28. Natarajan A, Samadian S, Clark S. Coronary artery bypass surgery in elderly people. Postgrad Med. 2007 Mar;83(977):154-8.

29. Hedeshian MH, Namour N, Dziadik E. Does increasing age have a negative impact on six-month functional outcome after coronary artery bypass? Surgery. 2002;12(1):239-44. 